\title{
Conditions required for binocular rivalry suppression
}

\author{
Alan W. Freeman and David F. Li \\ University of Sydney, Lidcombe, New South Wales, Australia
}

\begin{abstract}
When the two eyes are presented with incompatible stimuli, the two monocular stimuli are seen alternately in a never-ending cycle. It is now widely accepted that the neural processes underlying this phenomenon, binocular rivalry, are distributed across a number of cortical stages. It is not clear, however, where binocular rivalry is initiated. We performed two experiments whose aim was to clarify this issue. In the first experiment, rivalry was induced, and brief test stimuli were delivered to an eye while its inducing stimulus was either dominant or suppressed. Sensitivity to a test stimulus with features similar to those of the suppressed inducing stimulus was reduced only when the test was presented to the eye whose inducing stimulus was suppressed. This indicates that suppression of a monocular channel is a prerequisite for binocular rivalry suppression. The second experiment showed that to induce rivalry, local interocular stimulus incompatibilities were necessary and that conflicting global percepts were not sufficient. These results suggest that low-level visual processes are required for the initiation of binocular rivalry.
\end{abstract}

Binocular rivalry has become a popular tool for the study of visual perception because it produces a changing percept in the presence of a constant stimulus. The study of rivalry has, however, been accompanied by a controversy about the location in the visual pathway at which the suppressive effects of rivalry are exerted. The controversy can be summarized in two hypotheses about the mechanism of binocular rivalry suppression. Eye suppression holds that channels from one eye are suppressed and that any stimulus to the suppressed eye is reduced in visibility. This hypothesis is backed up by psychophysical evidence (Blake \& Fox, 1974b; Blake, Westendorf, \& Overton, 1980; Chen \& He, 2004; Nguyen, Freeman, \& Wenderoth, 2001; Wales \& Fox, 1970) and by functional magnetic resonance imaging on humans showing activity modulation in a monocularly driven cortical area (Tong \& Engel, 2001). There have also been recent reports that rivalry modulates eye-specific activity in the lateral geniculate nucleus (Haynes, Deichmann, \& Rees, 2005; Wunderlich, Schneider, \& Kastner, 2005).

The feature (or stimulus) suppression hypothesis, by contrast, states that it is a stimulus feature that is suppressed, regardless of the eye to which it is presented. Diaz-Caneja (1928) provided early evidence for this idea by presenting a semicircle adjacent to a grating to one eye and the mirror image of the arrangement to the other eye. Subjects tended to see a full circle or a grating and were, therefore, combining part of the left-eye stimulus with part of the right-eye stimulus to produce a coherent percept. Using a similar experimental design, Kovács, Papathomas, Yang, and Fehér (1996) presented an array of green spots interspersed with red spots to one eye. They presented spots with reversed colors to corresponding locations for the other eye, so that each red spot rivaled a green spot. Subjects saw a single color more often than chance, indicating that stimuli with the same features tend to be suppressed together. More psychophysical evidence for feature suppression came from the finding of normal rivalrous alternation when the stimuli were switched rapidly between the eyes (Logothetis, Leopold, \& Sheinberg, 1996). Finally, magnetic resonance imaging (Fang \& He, 2005; Tong, Nakayama, Vaughan, \& Kanwisher, 1998) and single-neuron recordings (Leopold \& Logothetis, 1996; Sheinberg \& Logothetis, 1997; Wilke, Logothetis, \& Leopold, 2006) have shown activity representing the suppressed stimulus in several areas beyond the primary visual cortex.

These opposing hypotheses have been largely reconciled with the realization that binocular rivalry is a process distributed across a number of cortical stages (Blake \& Logothetis, 2002). Eye suppression represents rivalry at work precortically or in the primary visual cortex, whereas feature suppression results from rivalrous processes in the higher cortex. There are, however, unanswered questions in this proposal, which our first experiment was designed to address.

First, the depth of binocular rivalry suppression can be measured by dividing visual sensitivity in the suppressed state by that during dominance (Blake \& Camisa, 1979; Nguyen et al., 2001). Is it therefore possible to measure the depths of both eye suppression and feature suppression in the same experiment and to compare those

A.W. Freeman, a.freeman@usyd.edu.au 
depths? We have approached this question by predicting the sensitivity changes expected when eye and feature suppression act alone and then fitting these predictions to the observations.

Second, although previous studies have produced clear evidence in favor of eye suppression (Blake \& Fox, 1974b; Blake et al., 1980; Chen \& He, 2004; Nguyen et al., 2001), there is reason to believe that the experimental designs used may have led to an underestimation for feature suppression. Because the stimuli in those studies were orthogonal gratings, subjects could have made their decisions about which monocular stimulus was dominant from the perceived orientation at a single visual field location. Their judgments could, therefore, have relied on activity in neurons with small receptive fields, such as those in the primary visual cortex. Although feature suppression is envisaged as a property of higher cortical areas, the responses measured in the previous work could have depended entirely on activity at lower levels. Can experiments be designed that depend more heavily on responses requiring higher level neural activity, thereby increasing the likelihood of revealing feature suppression? Our aim was to answer this question by measuring binocular rivalry suppression with a test stimulus for which the user's task was a sophisticated form discrimination. The subject had to make comparisons across a sizable area of the visual field in order to successfully complete the experimental task. It could, therefore, be expected that the subject's response would depend on the high-level neural machinery that is assumed to be at work in feature suppression. Thus, the experiments here should have the power to demonstrate feature suppression if it was present.

The aims of the first experiment, therefore, were twofold: to measure the depth of eye and feature suppression within the same experimental design and to provide a stronger test for feature suppression than had previously been used. A description of this work has previously been published in abstract form (Freeman \& Li, 2004).

Given the general agreement that binocular rivalry is a process distributed across a number of cortical stages, it is natural to ask where rivalry is initiated. One possibility is that rivalry is initiated by inhibition between left- and right-eye-dominated cells in the lateral geniculate nucleus or in Layer 4 of the primary visual cortex. There are published models (Freeman, 2005; Wilson, 2003) that make this assumption. Another, distinct, possibility is that rivalry arises through competition between high-level neural representations and spreads to the rest of the network via feedback pathways. Evidence for top-down initiation comes from the tendency for rivalry to produce ecologically valid percepts, rather than piecemeal ones (DiazCaneja, 1928; Kovács et al., 1996; T. L. Watson, Pearson, \& Clifford, 2004). Given the evidence for both bottom-up and top-down initiation and no clear way to favor one or the other hypothesis, we undertook a second experiment directed at this question. The experiment presented one eye with an array of dots rotating in one direction and the other eye with dots rotating in the opposite direction. Decreasing the lifetime of the dots reduced local stimulus competition but did not remove the percept of rotation.
The aim of this second experiment was to find out whether reducing dot lifetime would decrease binocular rivalry.

\section{EXPERIMENT 1}

\section{Predictions of the Hypotheses}

Figure 1A shows the stimuli used. Binocular rivalry was induced with a star-like pattern to one eye and a circle to the other. Adult human subjects viewed these stimuli and triggered a brief test stimulus when the right eye's inducing stimulus was dominant (on some runs) or suppressed (on the other runs). The test consisted of two adjacent lobed semicircles, and the subject's task was to indicate which semicircle had more lobes. The measurement of feature suppression places two requirements on the test stimulus. First, the test stimulus must be similar in features to one inducing stimulus, but not to the other. The mean threshold amplitude across all subjects was $5.8 \%$ of circle radius and is illustrated in Figure 1B; at threshold, the test appears similar in features to the circular rivalryinducing stimuli. More quantitatively, the angle between the threshold test and the circular inducing stimuli was measured at each point around their circumferences. The mean angular difference between them was $7^{\circ}$, whereas the difference between the test and the star-like stimuli was $83^{\circ}$.
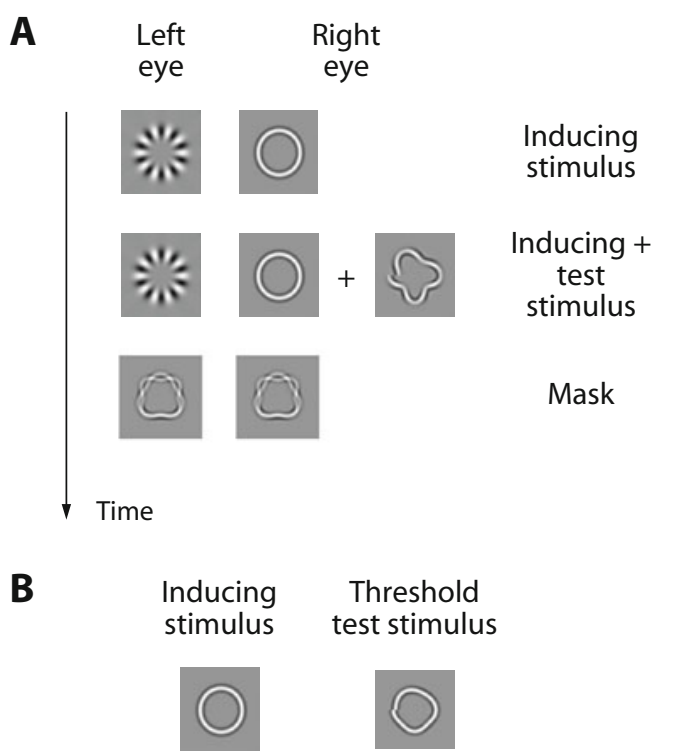

Figure 1. (A) Binocular rivalry was induced by presenting a star-like stimulus to one eye and a circle to the other. Subjects triggered a brief test stimulus to the right eye in some cases, or to both eyes in the remaining cases; only monocular presentation is illustrated here. The test stimulus was triggered when the right eye's inducing stimulus was dominant, on some runs, or suppressed, on the remaining runs. The test was followed by a mask. The subject's task was to indicate which semicircle in the test stimulus contained more lobes. The amplitude of the lobes was set at a level yielding $80 \%$ correct responses. (B) The figurine on the right side shows a test stimulus with lobe amplitude equal to the mean threshold across subjects. As was required by the experimental design, the stimulus had features similar to those of one of the inducing stimuli. 
The second requirement for the test stimulus is that the subject's task should involve feature discrimination. By feature, we mean a stimulus form that requires spatial pooling of local orientation information for its discrimination. There have been several studies indicating that responses to lobed circles fall into this category. First, Hess, Wang, and Dakin (1999) asked subjects to discriminate lobed circles from unlobed ones in two cases: (1) intact stimuli and (2) stimuli cut into four randomly arranged pieces. Discrimination was significantly poorer in the second case, indicating that the judgment was not based on spatially localized cues. They therefore concluded that the discrimination of lobed circles is more global than local, implicating neural mechanisms with large receptive fields. Second, Gallant, Braun, and Van Essen (1993) recorded single-neuron activity in a cortical area, V4, in which receptive fields are larger than those in the primary visual cortex, indicating that they spatially pool orientation information. They found a significant subpopulation of cells that responded better to concentric patterns than to rectilinear gratings. Finally, Wilkinson et al. (2000) used magnetic resonance imaging in humans to show that cortical area V4 is more strongly activated by lobed circle stimuli than by rectilinear gratings.

The test stimulus in our experiment was presented to the eye viewing the circular inducing stimulus, to the eye viewing the star-like stimulus, or to both eyes. The predictions of the eye suppression and feature suppression hypotheses for these three cases are derived from the following definitions.

Eye suppression is a loss of sensitivity to stimuli delivered to a specific eye. The loss does not depend on the type of stimulus, and there is no sensitivity loss for stimuli delivered to the fellow eye.

Feature suppression is a loss of sensitivity to stimuli with specific features. The loss does not depend on the eye to which the stimulus is delivered, and there is no sensitivity loss for stimuli with differing features.

The predictions are shown in Figure 2. Consider eye suppression first. The left side of its graph shows the case in which the test is presented to the eye viewing the circle, as illustrated by the figurines at the bottom of the figure. When the circle is dominant (open symbols), the tested eye is dominant, and the hypothesis predicts that sensitivity is high. But when the star is dominant (filled symbols), the tested eye is suppressed, and sensitivity is therefore reduced. The sensitivities are reversed for the data points in the middle of the graph, where the test is presented to the eye viewing the star. At the right of the graph, the test is presented to both eyes. Form discrimination sensitivity resulting from binocular presentation is only a little higher than that obtained with monocular viewing (Cagenello, Arditi, \& Halpern, 1993), and the binocular advantage is presumably even less when one eye is suppressed. Sensitivity in this case is therefore assumed to be equal to that of the dominant eye and is high regardless of which eye is dominant. The predictions of the feature suppression hypothesis also follow from its definition. When the test stimulus shares features with the dominant inducing stimulus, sensitivity is high regardless of which eye is tested.

\section{Eye Suppression Hypothesis}

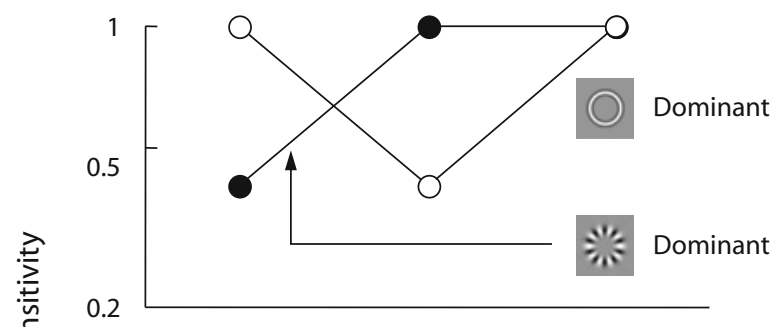

Feature Suppression Hypothesis

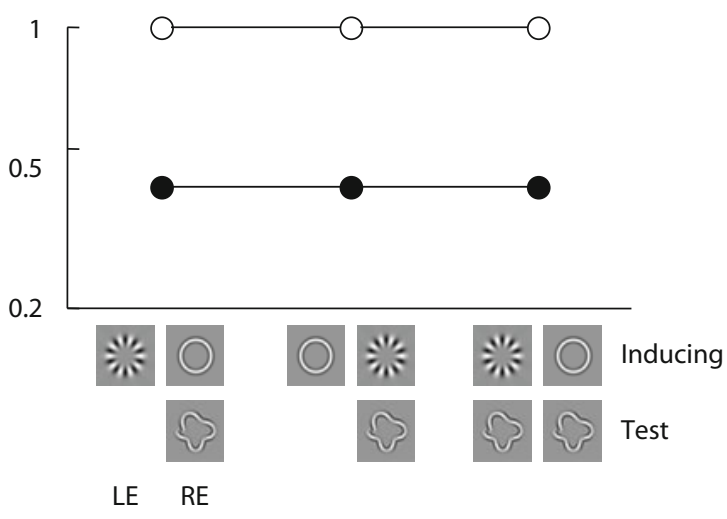

Stimulus

Figure 2. Predictions of the hypotheses. The figurines on the horizontal axis indicate the three cases tested. For each case, lefteye (LE) and right-eye (RE) stimuli are shown on the left and right, respectively. Test stimuli were delivered to the eye viewing the circular inducing stimulus, the eye viewing the star-like stimulus, or both. Open circles indicate predictions when the circular inducing stimulus is dominant; filled circles indicate dominance of the star. According to the eye suppression hypothesis, sensitivity is high when the test is presented to the dominant eye and is reduced otherwise. The feature suppression hypothesis gives differing predictions: Sensitivity is high when the test has features similar to those of the dominant inducing stimulus. The plotted data were calculated by assuming a suppression depth, $d$, equal to $0.4 \mathrm{log}$ units, for the purposes of illustration.

Sensitivity is lowered when the test stimulus has features like those of the suppressed inducing stimulus. These predictions are shown in the lower part of Figure 2.

A factor that could potentially alter these predictions is pattern-specific adaptation. Blakemore and Campbell (1969) showed that prolonged viewing of a grating reduces contrast sensitivity on a subsequent presentation of the grating. In binocular rivalry, a constant spatial pattern is presented to each eye over an extended period of time. Might dominance of a pattern result in more patternspecific adaptation than does suppression, changing the predictions of Figure 2? There was reason to think that adaptation would have only a minor influence in our experiment. Blake and Fox (1974a) showed that patternspecific adaptation produces the same sensitivity loss during constant viewing and during rivalry, despite the intermittent suppression of the adapting pattern in rivalry. It has recently been found that this conclusion must be 
modified when low-contrast adapting patterns are used (Blake, Tadin, Sobel, Raissian, \& Chong, 2006), but the latter finding is not relevant here, because we used highcontrast inducing stimuli.

\section{Method}

Subjects. There were 3 experimental subjects, 1 of them female and 2 male. They were 28 to 36 years of age, with normal visual acuity and a binocular disparity threshold of less than $1 \mathrm{~min}$. One subject (D.F.L.) was an author, and the other 2 were unaware of the aims or results of the experiments. The naive subjects gave their informed consent to their participation and were reimbursed for their time and travel expenses. All 3 subjects were highly experienced psychophysical observers.

Stimuli. Left-eye stimuli were presented on the left side of a computer screen, right-eye stimuli on the right side; the two views were separated with a septum, and the optical distance from screen to eye was $1.14 \mathrm{~m}$. The subjects used a mirror stereoscope and on-screen borders to fuse the monocular stimuli. All the stimuli were monochrome $\left(x=0.27, y=0.26\right.$, background luminance $\left.=26 \mathrm{~cd} / \mathrm{m}^{2}\right)$; spatial resolution was $1.1 \mathrm{~min}$ per pixel, and the video frame duration was $15 \mathrm{msec}$.

To produce the undistorted circles (as shown in Figure 1), luminance was set to the fourth derivative of a Gaussian function of radial distance, where the mean and standard deviation of the Gaussian were $r_{0}=0.67^{\circ}$ and $0.07 r_{0}$, respectively. Lobed circles were produced by using a variable radius, $r$, with $r-r_{0}$ a sinusoidal function of the angle between the radius and the horizontal. The circle-like test stimuli consisted of two lobed semicircles. One semicircle had 2 lobes, the other had 1.5 lobes, and lobe amplitude was the same for the two semicircles. The threshold variable was lobe amplitude, defined as a fraction of the radius $r$. Star stimuli were constructed by modulating luminance as a sinusoidal function of distance around a circle. The frequency of the sinusoid was set so that there were 10 arms. The star-like test stimulus (shown in Figure 4) contained a half-star with more than 5 arms and a second half-star with fewer than 5; the number of arms totaled 10 . The threshold variable was arm imbalance, defined as one tenth of the difference in arm number between the two half-stars.

The final step in constructing all the stimuli ensured that interocular stimulus differences were restricted to an annulus: Stimuli were multiplied by a Gaussian function of radial distance, where the Gaussian's mean and standard deviation were $r_{0}$ and $0.21 r_{0}$, respectively. The contrast at the points of highest luminance in the inducing and test stimuli was 0.45 when the circle-like test was used and 0.9 for the experiment employing the star-like test.

Procedure. On each trial of a dominance run, the subject waited until the right eye's inducing stimulus was exclusively visible. The subject then pressed a key, triggering an immediate test stimulus to the right eye or both eyes. Suppression runs were identical, except that tests were triggered when the left eye's inducing stimulus became visible. Rivalry was robust, in that the subjects did not have to wait for more than a few seconds for the required rivalry phase. When the test consisted of lobed semicircles, it was added to the inducing stimulus for $120 \mathrm{msec}$. The two-lobed semicircle in the test could be in either the upper or the lower position with equal probability, and the subject's task was to indicate the position of this semicircle. Lobes were randomly rotated around the circumference of each semicircle from trial to trial in order to prevent judgments based on small portions of the stimulus. When the tested eye's inducing stimulus was suppressed, the test tended to make it dominant; the test was therefore immediately followed by a mask in order to minimize cues arising from the dominance state. The mask consisted of a three-lobed circle added to a four-lobed circle, both with random orientation.

The other test stimulus, consisting of adjacent half-stars, replaced the inducing stimulus of one or both eyes for $120 \mathrm{msec}$. Replacement was used instead of addition, because the latter technique reduced sensitivities to the extent that they became difficult to measure. The subject's task in this case was to indicate the location of the half with more arms. Arm location within each half-star was randomized from trial to trial in order to eliminate local cues. The test was again followed immediately by a mask, which consisted of the sum of two stars. One star in the mask had the arm spacing of one of the half-stars in the test, and the other star had the spacing of the other half-star; arm location in the mask was randomized.

The subjects were provided with auditory feedback when the response was incorrect, and the threshold variable (lobe amplitude or arm imbalance) was varied from trial to trial, using a Quest procedure (A. B. Watson \& Pelli, 1983), to obtain $80 \%$ correct responses. The $80 \%$ criterion was used in both dominance and suppression runs. Each run was 25 trials long, and at least eight runs were recorded for each combination of the experimental parameters. The parameters were perceptual status (dominance or suppression) and the inducing stimulus to the tested eye(s) (circle, star, or both). The six parameter combinations were randomized in order, except that dominance runs alternated with suppression runs. Stimulus timing and threshold estimation were managed with the Psychophysics Toolbox (Brainard, 1997; Pelli, 1997).

Data analysis. Empirical data were fitted with the following equation: sensitivity $=d_{1} e+d_{2} f+d_{3}+\varepsilon$, where $e$ and $f$ are the predictions of the eye and feature suppression hypotheses, respectively, as shown in Figure 2. The $d$ s are regression coefficients, and $\varepsilon$ is residual error. The equation was first fitted to the logarithmic data from individual subjects. These data were then normalized by subtracting the constant term $d_{3}$ from them. Finally, the sensitivity equation was fitted to the combined normalized data from all the subjects; in this case, the regression coefficient $d_{3}$ was identically zero and was, therefore, omitted.

\section{Results}

Circular test stimulus. Empirical data from a single subject are shown in Figure 3A. The vertical axis shows sensitivity, calculated as the reciprocal of lobe amplitude threshold. Error bars, obtained from the eight or more sensitivities measured at each point, give $95 \%$ confidence intervals. A total of 3 subjects were tested on the task, and their results are shown in Figure 3B. The data have been normalized, as described in the Method section, to allow comparisons across subjects. The empirical data appear to match the predictions of the eye suppression hypothesis well and to fit poorly with the feature suppression predictions. To check on this conclusion, we fitted a regression equation, sensitivity $=d_{1} e+d_{2} f+\varepsilon$, to the logarithmic data. Figure 2 provides $e$ and $f$, which are the predictions of the eye and feature suppression hypotheses, respectively. The suppression depths $d_{1}$ and $d_{2}$ are the least-squares regression coefficients, and $\varepsilon$ is residual error. The coefficients, along with their $95 \%$ confidence intervals, were $d_{1}=0.394 \pm 0.058$ and $d_{2}=-0.008 \pm 0.047$. The first coefficient indicates that eye suppression produces a sensitivity loss of about $0.4 \log$ units, consistent with previous findings (Blake \& Camisa, 1979; Nguyen, Freeman, \& Alais, 2003; Nguyen et al., 2001). Although the sensitivity loss due to eye suppression is significantly different from zero $\left(p<10^{-10}\right.$ ), the confidence interval for $d_{2}$ includes zero, consistent with an absence of feature suppression.

Star-like test stimulus. The initial evidence favors the idea of eye suppression at the expense of feature suppression. We wanted to make sure, however, that the evidence was not biased by the choice of test stimulus. We therefore repeated the experiment, with the star as test stimulus. On 
A

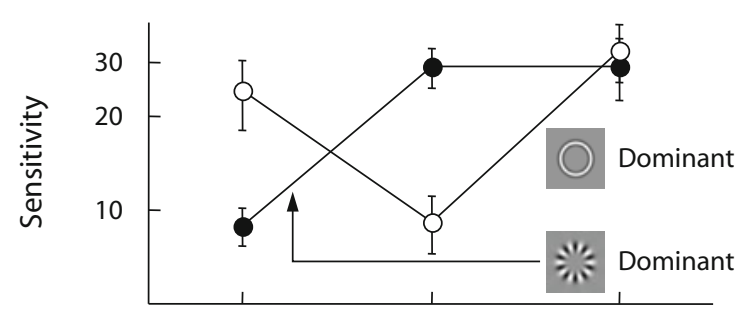

B

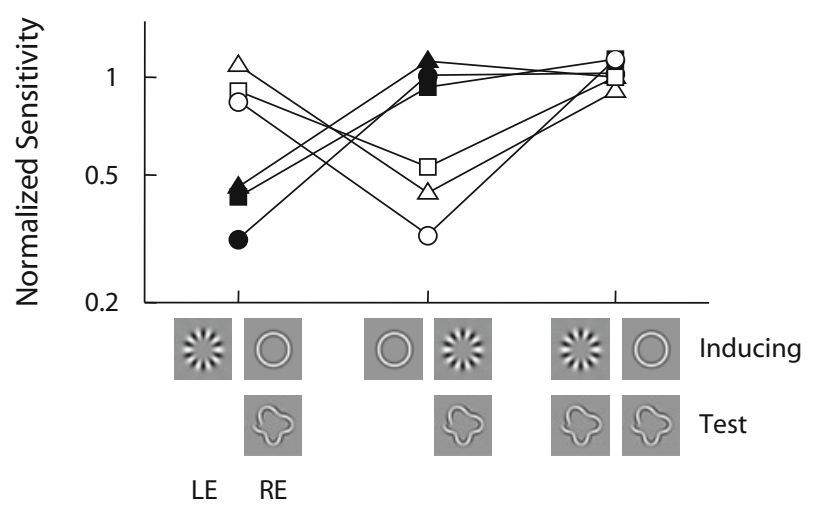

Stimulus

Figure 3. (A) Data from 1 subject, in the same format as that used in Figure 2. Sensitivity is calculated as the reciprocal of threshold lobe amplitude. Error bars show the $\mathbf{9 5 \%}$ confidence intervals. (B) Normalized data from 3 subjects. The data match well with the predictions of the eye suppression hypothesis and poorly with those of feature suppression. LE, left eye; RE, right eye.

any presentation of the test, there were more arms in one half of the stimulus and correspondingly fewer in the other half, as is shown in Figure 4A. The subject's task was to indicate the half with more arms. The threshold variable, the arm imbalance, was adjusted to obtain $80 \%$ correct responses. As is shown at the bottom of Figure 4A, the test stimulus at threshold appeared very similar to, and therefore shared features with, the star-like inducing stimulus. Data from a single subject are shown in Figure 4B, and normalized data from all 3 subjects are provided in Figure 4C.

Although these data have clear similarities to the predictions of the eye suppression hypothesis, there is also a difference. The mean sensitivity for the leftmost points is less than that for the points plotted in the middle of the graph. This trend upward is probably due to patternspecific adaptation. The points on the left were obtained when a test stimulus was presented to an eye viewing a similar inducing stimulus with substantial overlap of the arms in the inducing and test stimuli and are, therefore, subject to adaptation. The points in the middle are also subject to adaptation, but in this case, it is interocular, which is less than intraocular adaptation (Blakemore \& Campbell, 1969). Regardless of whether the adaptation is intra- or interocular, the adaptation effect applies to both rivalry dominance and suppression (Blake \& Fox, 1974a) and, therefore, has little or no effect on suppression depth. Fitting of the sensitivity equation to the combined data yielded $d_{1}=0.294 \pm 0.049$ and $d_{2}=0.018 \pm 0.040$. The confidence interval for the feature suppression coefficient $d_{2}$ includes zero, and the results obtained with the starlike test stimulus therefore correspond with those of the circle-like test: The data match the predictions of the eye suppression hypothesis well and are a very poor match with the feature suppression predictions.

\section{Discussion}

We have found that sensitivity is lower for stimuli presented to an eye during its suppression phase than for stimuli presented during dominance and that sensitivity is lowered for differing forms of the test stimulus. This agrees with the finding that changes in the orientation or spatial frequency of the suppressed stimulus can go undetected during rivalry (Blake \& Fox, 1974b; Nguyen et al., 2001). Conversely, although the experiments were designed to reveal the presence of feature suppression, we found clear evidence against it. Previous authors have detected the presence of feature suppression. How is this discrepancy resolved? The clearest evidence for feature suppression has come from Logothetis et al. (1996), who swapped rivalrous stimuli between the eyes three times a second but found dominance intervals that spanned several swaps. They also flickered their stimuli at a frequency of $18 \mathrm{~Hz}$, and it has been shown subsequently that the flicker plays an important role in producing normal dominance intervals during the swapping experiment (S.-H. Lee \& Blake, 1999). Wilson (2003) modeled this behavior and showed that flicker shifted coherent activity from the monocular stage of his model to the binocular stage, making it more like feature rivalry. It seems, therefore, that feature suppression is seen if the usual mutual inhibition between monocularly driven cells is prevented.

Another study providing evidence for feature suppression is that of O'Shea and Crassini (1981). They induced binocular rivalry with orthogonal gratings and briefly changed the orientation of one eye's grating while it was either dominant or suppressed. Subjects were required to signal in which direction the grating was changed, and the duration of change was adjusted to achieve $75 \%$ correct choices. Suppression of the reoriented grating elevated thresholds when the new grating orientation was close to that of either of the inducing orientations but did not change thresholds for orientation shifts between $20^{\circ}$ and $70^{\circ}$. This result contravenes one of the requirements of eye suppression - namely, that suppression depth be independent of the stimulus features used to test that depth. It should be noted, however, that O'Shea and Crassini used high-contrast (0.75) gratings and that the reorientation of one grating during suppression would, therefore, have had a powerful suppression-breaking effect. They did not use a posttest mask to reduce cues resulting when the previously suppressed eye became dominant. It could be, therefore, that the dependence of thresholds on orientation change resulted from the ability of the change to break suppression. 
A

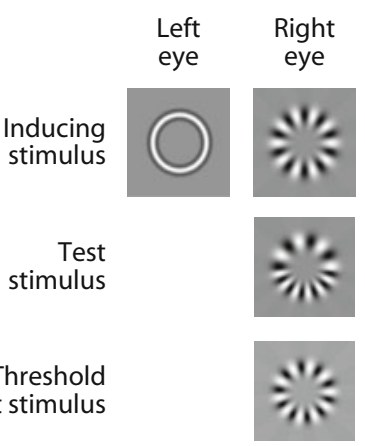

B
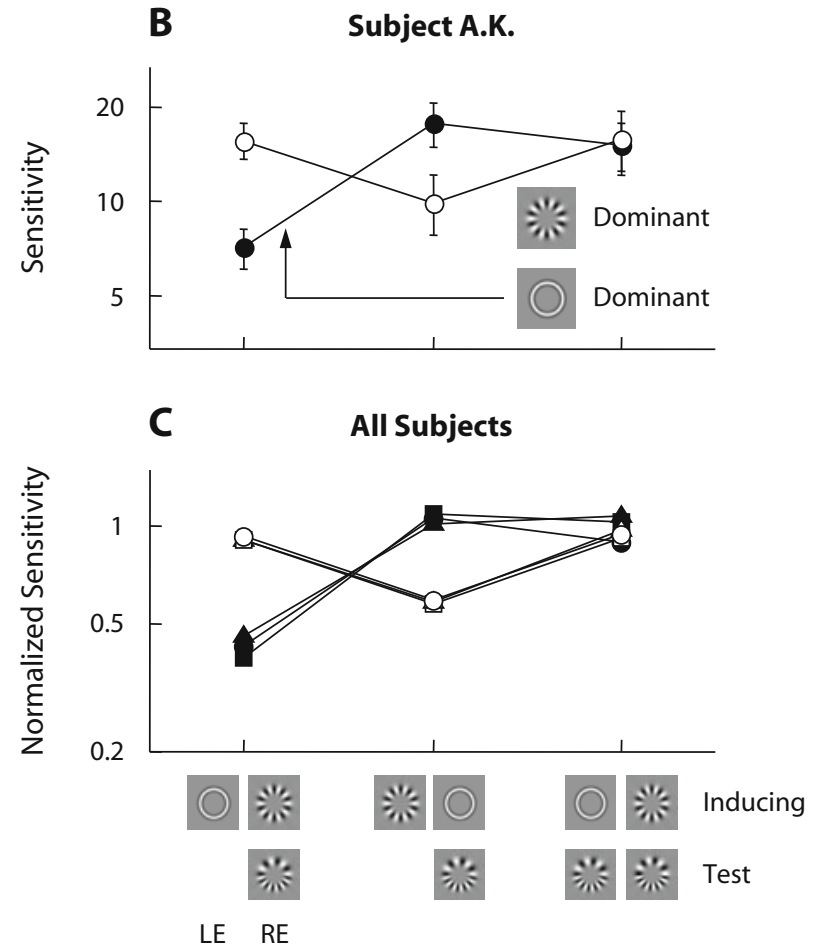

Stimulus

Figure 4. (A) As before, rivalry was induced with a star and circle. The test in this case consisted of adjoining half-stars, one of which had more arms than did the other. The subject's task was to indicate the location of the half-star with more arms. The mean arm imbalance threshold across all subjects was $7.2 \%$; the threshold test stimulus with this imbalance is shown. At threshold, the test stimulus had features similar to just one of the inducing stimuli. (B) Data from 1 subject. Sensitivity is calculated as the reciprocal of threshold arm imbalance, and error bars give the $95 \%$ confidence intervals. (C) Normalized data for all 3 subjects. Again, the data correspond well with the predictions of the eye, but not the feature, suppression hypothesis. LE, left eye; RE, right eye.

There are two other studies whose results may appear to favor the feature suppression hypothesis. T. L. Watson et al. (2004) used stimuli representing walking humans visible only through lights attached to their joints. When one walker was presented monocularly and a walker moving in the opposite direction was presented to the other eye, robust rivalry between the walkers ensued. Given the high-level perceptual grouping required to interpret a set of moving lights as a walker, this suggests that feature suppression is at work. However, a control experiment in which walkers were split between the eyes yielded weak rivalry, indicating that eye rivalry was the major effect. Watson et al. interpreted their results as showing that rivalry is exerted at a low level but is biased by top-down feedback favoring an ecologically plausible percept. The same interpretation presumably applies to the coherent percept obtained during simultaneous color rivalry at multiple visual field locations (Kovács et al., 1996). It seems, though, that at least some top-down biases are small, relative to bottom-up factors, because subjects have very little success when asked to consciously maintain one rivalrous percept at the expense of the other (Meng $\&$ Tong, 2004).

The results of our experiment and previous studies can be interpreted as follows. Although the evidence for feature suppression is clear, the present study shows that binocular rivalry suppression is eye specific: Test stimuli must be delivered to the eye whose inducing stimulus is suppressed if a loss of sensitivity is to be revealed. The two sets of data can be reconciled by assuming that eye suppression occurs before feature suppression. In other words, binocular rivalry is initiated by mutual inhibition between monocularly driven cells, and rivalry spreads from there along the visual pathway to areas containing binocularly driven cells. Burkhalter and Van Essen (1986) have shown that neurons in the visual cortex higher than the primary area are almost exclusively binocular. The simplest interpretation of the data, therefore, is that binocular rivalry originates in the primary visual cortex and spreads from there to higher cortical areas. Feature suppression, on the other hand, is revealed only by disabling eye suppression or as a top-down bias secondary to the effects of eye suppression.

\section{EXPERIMENT 2}

The evidence that binocular rivalry suppression is eye specific suggests that in the processing of signals leading to binocular rivalry, eye suppression comes before feature suppression. This, in turn, suggests that interocular conflict initiates the binocular rivalry process. The aim of our second experiment was to test the converse hypothesis - namely, that rivalry does not occur without interocular conflict. To do this, we used arrays of randomly placed dots rotating about a common center. Each dot had a lifetime: An infinite lifetime produced rigid rotation, and shorter lifetimes produced more random motion and a lessened rotation signal. Dots rotating in one direction were presented to one eye, and those rotating in the opposite direction to the other eye. Reducing dot lifetime lowers the probability that left-eye dots representing one rotation direction move in the direction opposite to right-eye dots at the corresponding visual location and, therefore, decreases coherent interocular conflict. The expectation, therefore, was that reducing dot lifetime would also reduce the duration of binocular rivalry. 


\section{Method}

The visual stimuli consisted of dot patterns, as illustrated on the left of Figure 5. The pattern for each eye was a collection of randomly distributed white dots (with a luminance of $48 \mathrm{~cd} / \mathrm{m}^{2}$ ) against a black background $\left(0.45 \mathrm{~cd} / \mathrm{m}^{2}\right)$. The dots were centered within a square border, inner diameter $2.67^{\circ}$, provided as an aid to binocular fusion. There were 30 dots contained in a circle with a diameter of $1.33^{\circ}$, resulting in a mean density of $21.5 \mathrm{dots} / \mathrm{deg}^{2}$. Each dot consisted of a single pixel, subtending $1.1 \mathrm{~min}$. The dots rotated around the center of the circle at a speed of one revolution every $7 \mathrm{sec}$. A variety of dot densities and speeds were tested in a pilot experiment. The values settled on were those that produced coherent binocular rivalry about half the time when the dot lifetime was infinite. The lifetime of a dot was defined as the duration, or the number of video frames, of continuous rotation before the dot was randomly relocated. The longest lifetime was infinite, for which the dot pattern rotated rigidly. The shortest lifetime was one video frame, with a duration of $15 \mathrm{msec}$. At this lifetime, all the dots were randomly relocated on each frame, resulting in no coherent rotation signal in the stimulus.

We used three experimental protocols. First, to measure binocular rivalry, the stimulus consisted of a rotating dot pattern presented to one eye and a second pattern rotating in the other direction, presented to the other eye. Dot placement in one pattern was independent of that in the other pattern. The clockwise pattern was presented to the left eye, and the counterclockwise pattern to the right eye, as shown in Figure 5A. Rivalry was measured as the duration for which coherent motion, either counterclockwise or clockwise, was perceived. The subjects indicated their perceptual status by pressing one of three keys for counterclockwise, mixed, or clockwise percepts, respectively. The duration of each keypress was measured. Observations lasted $60 \mathrm{sec}$, and there were 10 observation periods for each dot lifetime.

The second experimental protocol determined the strength of the motion percept in the absence of rivalry. Identical rotating dot patterns were presented to the two eyes, as illustrated in Figure 5B, for $1 \mathrm{sec}$ on each trial. The rotation directions, either counterclockwise or clockwise, were equally probable on each trial. There were 60 trials in each experimental run, and each trial was randomly assigned one of six dot lifetimes, with the constraint that each lifetime was used on 10 trials. There were at least 10 runs, so that each dot lifetime was presented on at least 100 trials. The subject's task on each trial was to indicate the rotation direction by pressing one of two keys.

The third experimental protocol measured monocular rivalry. The stimulus to the left eye consisted of two intermingled dot patterns, with one rotating in the direction opposite to that of the other. The right-eye stimulus was identical to the left one, as shown in Figure $5 \mathrm{C}$. There were 60 dots per eye, half of them rotating clockwise and the remainder rotating counterclockwise. This protocol was otherwise the same as that used to measure binocular rivalry.

\section{Results}

The horizontal axes in Figure 5 show dot lifetime in terms of both video frames and time, and error bars show the $95 \%$ confidence intervals. Panel A of the figure shows the probability of coherent perception for the 3 subjects. This probability was calculated by adding the duration for which clockwise rotation was perceived to the duration for a counterclockwise percept and dividing by total viewing time. The 3 subjects had similar results, in that the probability of coherent perception was zero for the shortest lifetime and increased as a function of dot lifetime. An ANOVA showed that probability depended on lifetime [repeated measures $F(4,8)=9.7, p=.004$ ].

There are two possible explanations for the lack of binocular rivalry at short lifetimes. First, the rotation signal in each of the dot patterns may have been too weak to
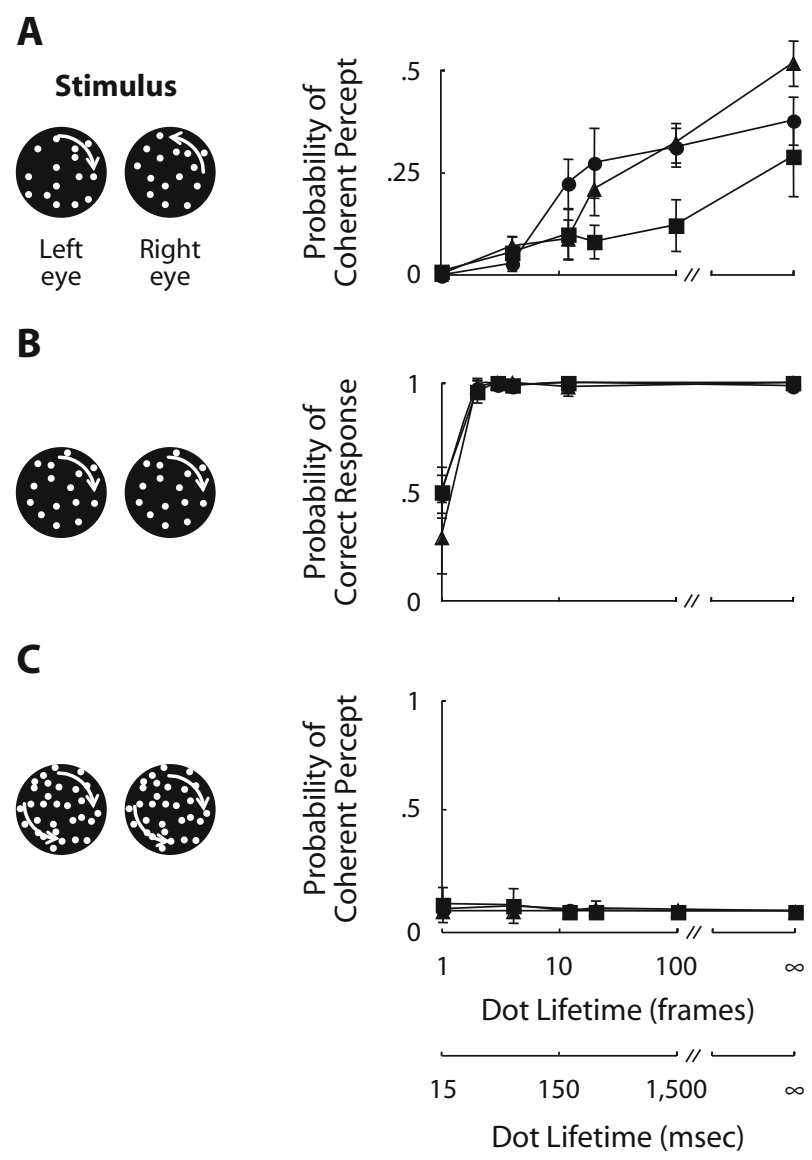

Figure 5. Stimuli were random-dot patterns rotating about a common center. Dot lifetime, shown on the horizontal axis, ranged from one video frame to infinite. Three subjects are represented on each graph, and error bars give $95 \%$ confidence intervals. (A) Binocular rivalry was induced with a pattern rotating clockwise for one eye and counterclockwise for the other eye. The vertical axis gives the fraction of viewing time for which the subject saw a single rotation direction. Rivalry is absent for the lowest dot lifetimes but becomes more frequent with increasing dot lifetime. (B) Dots were rotated in the same direction for both eyes. The vertical axis gives the probability that the subject could correctly discriminate the rotation direction. (C) Dots rotated in both directions in each monocular stimulus, and the two eyes were stimulated identically. Subjects indicated whether they saw a single rotation direction. Monocular rivalry is absent at all dot lifetimes.

induce rivalry. Second, the rotation signals may have been sufficiently strong but may have evoked insufficient local competition to produce rivalry. Panel B of Figure 5 rules out the first possibility. It shows the probability of correct discrimination of motion direction when the two eyes were presented with identical stimuli. For the shortest lifetime (one video frame), there was no coherent rotation signal in the stimulus, and correspondingly, the probability of a correct choice was close to chance (mean probability $=$ .43 , ranging from .29 to .50 ). For the remaining lifetimes, discrimination was close to perfect (mean probability $=$ .99 , ranging from .96 to 1.00 ). The conclusion is that when a rotation signal is present, it is strong enough to allow discrimination of its direction. 
We are left with the hypothesis that binocular rivalry is evoked only if there is enough local interstimulus competition; competing percepts are not sufficient to produce rivalry. The differing stimuli in Figure 5A were delivered to different eyes. Is it possible for competing stimuli to evoke rivalry even when they are delivered to the same eye? Previous work has shown that this form of rivalry, termed monocular rivalry, is experienced when crossed gratings with differing colors are presented to the same eye (Campbell, Gilinsky, Howell, Riggs, \& Atkinson, 1973). Figure 5C shows that there was no monocular rivalry with the stimuli we used. The vertical axis in the figure gives the duration for which a single direction of rotation was seen, divided by total stimulation time. The response is essentially zero (mean probability $=.01$, ranging from 0 to .03), indicating an absence of monocular rivalry. We conclude from this experiment that for rivalry to occur, the local stimulus conflict must be interocular.

\section{Discussion}

Our subjects were able to discriminate the rotation direction in the stimulus at all lifetimes for which the stimulus contained a rotation signal (Figure 5B). Given that the rotation was so clearly visible, one might expect vigorous competition between the stimuli when the rotation direction presented to one eye opposed that to the other eye. In fact, there was virtually no rivalry at low dot lifetimes. This result indicates that competing global percepts were not sufficient to produce rivalry; there was also a requirement on the stimulus structure at a more restricted spatial scale. As dot lifetime decreased, there was a corresponding reduction in the probability that left- and right-eye dots were moving in the opposite direction. Apparently, it was this reduction in local interocular competition that produced the absence of rivalry. The attempt to find monocular rivalry (Figure 5C) adds to this finding. With long dot lifetimes, two of the requirements for rivalry - local stimulus conflict and the potential for perceptual competition - were met. There was no rivalry, however, because the two eyes were stimulated identically. The conclusion from Experiment 2, therefore, is that one of the conditions required for binocular rivalry is local interocular stimulus conflict.

\section{GENERAL DISCUSSION}

We have shown two things in this article. First, a test stimulus presented during binocular rivalry loses visibility only when presented to the eye whose inducing stimulus is suppressed, regardless of similarities between the test and the suppressed inducing stimulus. Second, monocular stimuli producing competing percepts do not produce binocular rivalry unless there is also local interocular conflict between the stimuli. Thus, we have found two necessary conditions for binocular rivalry suppression: (1) eye suppression and (2) local interocular conflict.

Although these conditions are necessary for binocular rivalry suppression, we do not claim that they are sufficient for such suppression. For example, Alais and Parker (2006) induced rivalry between an expanding stimulus to one eye and a contracting stimulus to the other eye. They then required their subjects to discriminate between faces briefly presented to either the suppressed or the dominant eye. Discrimination thresholds were not significantly different for these two cases, indicating that rivalry between motions did not affect form stimuli presented to the suppressed eye. Conversely, face rivalry did not alter motion thresholds. It seems, therefore, that there is at least a third condition required for the production of binocular rivalry suppression - namely, that where there is a choice between form and motion, the test stimuli must be of the same kind as the inducing stimuli.

Given the dependence of binocular rivalry on neural processes at the input stages of the visual cortex, a parallel can be drawn between rivalry and other low-level phenomena producing perceptual selection. These other mechanisms include flash suppression, in which a stimulus suddenly presented to one eye gains perceptual dominance over a static stimulus to the other eye (Wolfe, 1984), and the involuntary attention-attracting effects of perturbing one of two superimposed stimuli (Reynolds, Alborzian, \& Stoner, 2003; Valdes-Sosa, Cobo, \& Pinilla, 2000). How do these low-level effects lead to perceptual selection? We have recently developed a neural model (Freeman, 2005) that helps to answer this question. In the model, dominance of one percept over another results from small activity differences between channels in the low-level visual cortex. Small differences early on then amplify through successive processing stages to produce large activity differences between channels in the higher cortex. This is a winner-takeall mechanism that has also been successfully applied to studies of attention (D. K. Lee, Itti, Koch, \& Braun, 1999) and object recognition (Riesenhuber \& Poggio, 1999). It will be interesting to discover whether this mechanism proves to be a widespread property of visual processing.

\section{AUTHOR NOTE}

We thank R. Balnave for comments on an earlier version of the manuscript. Correspondence concerning this article should be addressed to A. W. Freeman, School of Medical Sciences, University of Sydney, P.O. Box 170, Lidcombe, NSW 1825, Australia (e-mail: a.freeman@ usyd.edu.au).

Note-Accepted by the previous editorial team, when Thomas H. Carr was Editor.

\section{REFERENCES}

Alais, D., \& PARKer, A. (2006). Independent binocular rivalry processes for motion and form. Neuron, 52, 911-920. doi:10.1016/j .neuron.2006.10.027

BlaKe, R., \& CAMISA, J. (1979). On the inhibitory nature of binocular rivalry suppression. Journal of Experimental Psychology: Human Perception \& Performance, 5, 315-323. doi:10.1037/0096-1523.5.2.315

Blake, R., \& Fox, R. (1974a). Adaptation to invisible gratings and the site of binocular rivalry suppression. Nature, 249, 488-490. doi: $10.1038 / 249488 \mathrm{a} 0$

BlaKe, R., \& Fox, R. (1974b). Binocular rivalry suppression: Insensitive to spatial frequency and orientation change. Vision Research, 14, 687-692. doi:10.1016/0042-6989(74)90065-0

Blake, R., \& Logothetis, N. K. (2002). Visual competition. Nature Reviews Neuroscience, 3, 13-21. doi:10.1038/nrn701

Blake, R., Tadin, D., Sobel, K. V., Raissian, T. A., \& Chong, S. C. (2006). Strength of early visual adaptation depends on visual awareness. Proceedings of the National Academy of Sciences, 103, 4783-4788. doi:10.1073/pnas.0509634103 
Blake, R., Westendorf, D. H., \& Overton, R. (1980). What is suppressed during binocular rivalry? Perception, 9, 223-231. doi:10.1068/ p090223

Blakemore, C. [B.], \& CAmpbell, F. W. (1969). On the existence of neurones in the human visual system selectively sensitive to the orientation and size of retinal images. Journal of Physiology, 203, 237-260.

Brainard, D. H. (1997). The Psychophysics Toolbox. Spatial Vision, 10, 433-436. doi:10.1163/156856897X00357

Burkhalter, A., \& VAn Essen, D. C. (1986). Processing of color, form and disparity information in visual areas VP and V2 of ventral extrastriate cortex in the macaque monkey. Journal of Neuroscience, 6, 2327-2351

Cagenello, R., Arditi, A., \& Halpern, D. L. (1993). Binocular enhancement of visual acuity. Journal of the Optical Society of America A, 10, 1841-1848. doi:10.1364/JOSAA.10.001841

Campbell, F. W., Gilinsky, A. S., Howell, E. R., Riggs, L. A., \& AtKinson, J. (1973). The dependence of monocular rivalry on orientation. Perception, 2, 123-125. doi:10.1068/p020123

Chen, X., \& He, S. (2004). Local factors determine the stabilization of monocular ambiguous and binocular rivalry stimuli. Current Biology, 14, 1013-1017. doi:10.1016/j.cub.2004.05.042

Diaz-Caneja, E. (1928). Sur l'alternance binoculaire. Annales d'Oculistique, 165, 721-731. [Translated by Alais, D., O'Shea, R. P., Mesana-Alais, C., \& Wilson, I. G. (2000). On binocular alternation. Perception, 29, 1437-1445. doi:10.1068/p3017]

FANG, F., \& He, S. (2005). Cortical responses to invisible objects in the human dorsal and ventral pathways. Nature Neuroscience, $\mathbf{8}$, 1380-1385. doi:10.1038/nn1537

Freeman, A. W. (2005). Multistage model for binocular rivalry. Journal of Neurophysiology, 94, 4412-4420. doi:10.1152/jn.00557.2005

Freeman, A. W., \& Li, D. F. (2004, October). Binocular rivalry: Feature suppression requires eye suppression. Paper presented at the 34 th Annual Meeting of the Society for Neuroscience, San Diego.

Gallant, J. L., Braun, J., \& Van Essen, D. C. (1993). Selectivity for polar, hyperbolic, and Cartesian gratings in macaque visual cortex. Science, 259, 100-103. doi:10.1126/science.8418487

Haynes, J.-D., Deichmann, R., \& Rees, G. (2005). Eye-specific effects of binocular rivalry in the human lateral geniculate nucleus. Nature, 438, 496-499. doi:10.1038/nature04169

Hess, R. F., Wang, Y.-Z., \& Dakin, S. C. (1999). Are judgements of circularity local or global? Vision Research, 39, 4354-4360. doi:10.1016/ S0042-6989(99)00153-4

Kovács, I., PApathomas, T. V., YAng, M., \& Fehér, Á. (1996). When the brain changes its mind: Interocular grouping during binocular rivalry. Proceedings of the National Academy of Sciences, 93, 15508-15511.

Lee, D. K., ItTi, L., Koch, C., \& Braun, J. (1999). Attention activates winner-take-all competition among visual filters. Nature Neuroscience, 2, 375-381. doi:10.1038/7286

LEE, S.-H., \& BlAKE, R. (1999). Rival ideas about binocular rivalry. Vision Research, 39, 1447-1454. doi:10.1016/S0042-6989(98)00269-7

Leopold, D. A., \& Logothetis, N. K. (1996). Activity changes in early visual cortex reflect monkeys' percepts during binocular rivalry. $\mathrm{Na}$ ture, 379, 549-553. doi:10.1038/379549a0

Logothetis, N. K., Leopold, D. A., \& Sheinberg, D. L. (1996). What is rivalling during binocular rivalry? Nature, 380, 621-624. doi: $10.1038 / 380621 \mathrm{a} 0$

Meng, M., \& Tong, F. (2004). Can attention selectively bias bistable perception? Differences between binocular rivalry and ambiguous figures. Journal of Vision, 4, 539-551. doi:10.1167/4.7.2
Nguyen, V. A., Freeman, A. W., \& Alais, D. (2003). Increasing depth of binocular rivalry suppression along two visual pathways. Vision Research, 43, 2003-2008. doi:10.1016/S0042-6989(03)00314-6

Nguyen, V. A., Freeman, A. W., \& Wenderoth, P. (2001). The depth and selectivity of suppression in binocular rivalry. Perception \& Psychophysics, 63, 348-360.

O'Shea, R. P., \& Crassini, B. (1981). The sensitivity of binocular rivalry suppression to changes in orientation assessed by reaction-time and forced-choice techniques. Perception, 10, 283-293. doi:10.1068/ p100283

Pelli, D. G. (1997). The VideoToolbox software for visual psychophysics: Transforming numbers into movies. Spatial Vision, 10 437-442. doi:10.1163/156856897X00366

Reynolds, J. H., Alborzian, S., \& Stoner, G. R. (2003). Exogenously cued attention triggers competitive selection of surfaces. Vision Research, 43, 59-66. doi:10.1016/S0042-6989(02)00403-0

RieSENHUber, M., \& Poggio, T. (1999). Hierarchical models of object recognition in cortex. Nature Neuroscience, 2, 1019-1025. doi:10.1038/14819

Sheinberg, D. L., \& Logothetis, N. K. (1997). The role of temporal cortical areas in perceptual organization. Proceedings of the National Academy of Sciences, 94, 3408-3413.

Tong, F., \& ENGEL, S. A. (2001). Interocular rivalry revealed in the human cortical blind-spot representation. Nature, 411, 195-199 doi:10.1038/35075583

Tong, F., Nakayama, K., Vaughan, J. T., \& Kanwisher, N. (1998). Binocular rivalry and visual awareness in human extrastriate cortex. Neuron, 21, 753-759. doi:10.1016/S0896-6273(00)80592-9

Valdes-Sosa, M., Cobo, A., \& Pinilla, T. (2000). Attention to object files defined by transparent motion. Journal of Experimental Psychology: Human Perception \& Performance, 26, 488-505. doi:10.1037/0096-1523.26.2.488

WALES, R., \& Fox, R. (1970). Increment detection thresholds during binocular rivalry suppression. Perception \& Psychophysics, 8, 90-94.

Watson, A. B., \& Pelli, D. G. (1983). QUEST: A Bayesian adaptive psychometric method. Perception \& Psychophysics, 33, 113-120.

Watson, T. L., Pearson, J., \& Clifford, C. W. G. (2004). Perceptual grouping of biological motion promotes binocular rivalry. Current Biology, 14, 1670-1674. doi:10.1016/j.cub.2004.08.064

Wilke, M., Logothetis, N. K., \& Leopold, D. A. (2006). Local field potential reflects perceptual suppression in monkey visual cortex. Proceedings of the National Academy of Sciences, 103, 17507-17512. doi:10.1073/pnas.0604673103

Wilkinson, F., James, T. W., Wilson, H. R., Gati, J. S., Menon, R. S. \& Goodale, M. A. (2000). An fMRI study of the selective activation of human extrastriate form vision areas by radial and concentric gratings. Current Biology, 10, 1455-1458. doi:10.1016/S09609822(00)00800-9

WiLson, H. R. (2003). Computational evidence for a rivalry hierarchy in vision. Proceedings of the National Academy of Sciences, 100, 14499-14503. doi:10.1073/pnas.2333622100

Wolfe, J. M. (1984). Reversing ocular dominance and suppression in a single flash. Vision Research, 24, 471-478. doi:10.1016/00426989(84)90044-0

Wunderlich, K., Schneider, K. A., \& Kastner, S. (2005). Neural correlates of binocular rivalry in the human lateral geniculate nucleus. Nature Neuroscience, 8, 1595-1602. doi:10.1038/nn1554

(Manuscript received March 21, 2007; revision accepted for publication July 21, 2008.) 